ulated by at least fivefold, and 60 similarly down-regulated genes. Among those that increased are neuron-associated genes such as APP and neuronal protein 3.1, and genes associated with tissue remodeling (proteinases and inhibitors). Many of the affected genes are unannotated expressed sequence tags (ESTs), and these experiments will contribute to an understanding of their functions. Since differentiation of ES cells can be directed in several ways in culture, our studies will define a set of genes for each cell type. These gene sets may be involved in the determination and differentiation of embryonic cells in vivo.

Los, Gerrit

\section{Identification of genes differentially expressed in cisplatin-sensitive versus resistant tumour cells}

\author{
G. Los, A. Johnson, F. Yang, C. Berry \& S. Howell \\ UCSD Cancer Center, La Jolla, California 92093, USA
}

Acquired resistance to cDDP (cisplatin, a cytostatic drug) appears to be a multifactorial process and is likely to be the result of an altered gene expression pattern. The goal of this study is to profile such an expression pattern by identifying genes whose mRNA levels are differentially expressed in cDDP-sensitive cells (UMSCC10b) versus its resistant variant (UMSCC10b/Pt-S15). Using suppressive subtraction hybridization (SSH) and cDNA expression array technology, 400 clones were randomly selected and differentially screened using cDNA arrays, resulting in 2 different cDNA libraries: a UP library with 51 cDNA fragments (upregulated in cDDP-resistant variants) and a DOWN library with 16 fragments (downregulated in the cDDP-resistant variants). We positively identified $87 \%$ of the cDNA fragments in GenBank. To identify those mRNAs whose change in expression level was most closely linked to the degree of cDDP resistance, cDNA probes obtained from the parental UMSCC10b cells and four resistant UMSCC $10 b$ variants with different levels of resistance (two- to sixfold) were used to hybridize cDNA arrays containing the UP and DOWN libraries. Among the mRNAs analysed, only one demonstrated a clear association with the magnitude of resistance (cytochrome oxidase mRNA), all the other mRNAs showed increased levels in resistant variants, but these increases varied only slightly at the various levels of resistance. Given the function of most of the identified genes (encoding cytochrome oxidase, ribosomal protein 28S, elongation factor 1a, glyceraldehyde phosphate dehydrogenase, a-enolase, stathmin and HSP70), it appears that they mainly represent genetic downstream events, thus describing a resistant phenotype with increased level of metabolism, proliferation rate, protein synthesis and cytoskeletal function as well as activated signalling pathways.

\section{Lou, Xing Jian}

\section{Genotyping drug-metabolizing enzyme variation using microarrays}

\author{
Xing Jian Lou, Curtis Kautzer \& Elaine M ansfield
}

Pharmacogenomics, diaDexus, LLC, Santa Clara, California 95054, USA

Adverse drug reactions are the fourth-leading cause of death in the United States ${ }^{1}$. In addition, many patients are refractory to drug treatment or receive limited benefit from such therapy. DNA sequence polymorphisms have been found in genes encoding the drug-metabolizing enzymes (DMEs). In addition, variation in drug receptors and the genes that regulate DMEs can also have a role in differential drug response. Selected sequence polymorphisms correlate with an increased risk of drug toxicity or reduction in efficacy. It has become clear recently that each of us has his or her own 'individual fingerprint' of unique alleles encoded by DMEs and related genes. The identification of the fingerprints of variation in DMEs will become invaluable for the selection and optimization of drug therapy but requires development of robust, cost-effective parallel analysis of the sequence variants. To develop a platform for parallel analysis of polymorphism in DMEs, a microarray containing 42 mutations was constructed by printing amine-modified oligonucleotides on microscope slides pre-coated with amine-reactive groups. Cy3- or Cy5-labelled oligonucleotides complementary to wild-type or mutant alleles, as well as probes generated by multiplex PCR from genomic DNA of patients, were hybridized to the array. Different hybridization conditions were compared to increase the sensitivity and specificity of detection.

1. Lazarou, J. et al. JAMA 279, 1200-1205 (1998).

Lucito, Robert

\section{Application of representational microar- rays to genetic analysis of primary tumours}

\author{
Rob Lucito, Joe West, Andy Reiner, Joan Alexander, Clifford Yen, \\ Mariko Nakamura \& M ike Wigler
}

Cold Spring Harbor Laboratory, Cold Spring Harbor, New York, USA

We have explored using DNA microarrays for measuring gene copy number in cancer biopsies. The high nucleotide complexity of the human genome makes hybridization analysis of the entire genome problematic. Therefore, we have used the approach of making complexity-reducing representations ${ }^{1}$. Accordingly, the printed probes themselves are derived from simplified representations of the human genome, and are hybridized to representations of tumour and normal DNA. Data from pilot experiments indicate that this method can be used to detect amplifications and deletions in cancer specimens. The theoretical resolving power of these microarrays is on the order of $100 \mathrm{~kb}$ with a 30,000-member array, but higher resolving power is not excluded. In contrast to expression array analysis, only minute amounts of starting material (5,000 nuclei) are sufficient for sample preparation. Also, the starting material for analysis is DNA not RNA, which is easier to obtain from surgical specimens. The two methods should work best in concert; that is, combining copy-number analysis with expression analysis for the same tumours, enabling the user to more readily identify the primary defects in cancer cells. Presently we have produced a 2,000-member array that contains fragments of known sequence and mapping location as well as fragments cloned randomly from a representation. The copy number of many of these probes is known in respect to several tumours we have characterized by more conventional methods. Representations from these tumour pairs have been hybridized to these arrays and analysed. By querying the hybridization results we have identified the fragments known to be effected in these tumours. We are now confirming the results of the hybridizations by more accepted techniques. Several obstacles remain to creating a high-density genomic chip, including the assembly of a collection of probes, mapping them and developing the software tools to seamlessly analyse the data. Genomic chips have uses beyond cancer, including detection of polymorphisms in individuals and de novo germline rearrangements.

1. Lucito, R. et al. Proc. Natl Acad. Sci. USA 95, 4487-4492 (1998). 